\title{
Three-Axis Inductive Displacement Sensor Using Phase-Sensitive Digital Signal Processing for Industrial Magnetic Bearing Applications
}

\author{
Teemu Sillanpää*(D), Alexander Smirnov (D), Pekko Jaatinen (D), Jouni Vuojolainen (D), Niko Nevaranta (D), \\ Rafal Jastrzebski (D) and Olli Pyrhönen (D)
}

check for updates

Citation: Sillanpää, T.; Smirnov, A.; Jaatinen, P.; Vuojolainen, J.;

Nevaranta, N.; Jastrzebski, R.;

Pyrhönen, $\mathrm{O}$. Three-Axis Inductive

Displacement Sensor Using

Phase-Sensitive Digital Signal

Processing for Industrial Magnetic

Bearing Applications. Actuators 2021

10, 115. https://doi.org/10.3390/

act10060115

Academic Editor: Satoshi Ueno

Received: 29 April 2021

Accepted: 26 May 2021

Published: 31 May 2021

Publisher's Note: MDPI stays neutral with regard to jurisdictional claims in published maps and institutional affiliations.

Copyright: (c) 2021 by the authors. Licensee MDPI, Basel, Switzerland. This article is an open access article distributed under the terms and conditions of the Creative Commons Attribution (CC BY) license (https:// creativecommons.org/licenses/by/ $4.0 /)$.
School of Energy Systems, Lappeenranta-Lahti University of Technology LUT, Skinnarilankatu 34, 53850 Lappeenranta, Finland; alexander.smirnov@spindrive.fi (A.S.); Pekko.Jaatinen@lut.fi (P.J.); jouni.vuojolainen@lut.fi (J.V.); niko.nevaranta@lut.fi (N.N.); Rafal.Jastrzebski@lut.fi (R.J.); olli.pyrhonen@lut.fi (O.P.)

* Correspondence: teemu.sillanpaa@lut.fi
Abstract: Non-contact rotor position sensors are an essential part of control systems in magnetically suspended high-speed drives. In typical active magnetic bearing (AMB) levitated high-speed machine applications, the displacement of the rotor in the mechanical air gap is measured with commercially available eddy current-based displacement sensors. The aim of this paper is to propose a robust and compact three-dimensional position sensor that can measure the rotor displacement of an AMB system in both the radial and axial directions. The paper presents a sensor design utilizing only a single unified sensor stator and a single shared rotor mounted target piece surface to achieve the measurement of all three measurement axes. The sensor uses an inductive measuring principle to sense the air gap between the sensor stator and rotor piece, which makes it robust to surface variations of the sensing target. Combined with the sensor design, a state of the art fully digital signal processing chain utilizing synchronous in-phase and quadrature demodulation is presented. The feasibility of the proposed sensor design is verified in a closed-loop control application utilizing a 350-kW, 15,000-r/min high-speed industrial induction machine with magnetic bearing suspension. The inductive sensor provides an alternative solution to commercial eddy current displacement sensors. It meets the application requirements and has a robust construction utilizing conventional electrical steel lamination stacks and copper winding.

Keywords: digital signal processing; displacement measurement; inductive sensor; magnetic bearings; rotor system

\section{Introduction}

Many industrial processes are based on pumping or compressing liquids or gases. Such processes include, for example waste-water purification plants based on aeration compression, heating, ventilation, air conditioning and refrigeration (HVACR) systems based on liquid chiller compression and sub-sea gas compression units helping to utilize the full potential of subsea gas fields. A common factor for these processes is that the actuator, for example, a compressor, pump or blower, benefits from the use of very high rotational speeds of up to several hundred thousand rotations per minute. The high rotational speed places extra demands on the rotor suspension. Non-contact active magnetic bearings (AMBs), as a suspension solution, provide oil-free, frictionless and maintenance-free operation compared to competing bearing technology such as roller or journal bearings [1]. The low-efficiency gearbox between the electrical machine and actuator can be removed by integrating the frequency converter driven high-speed electrical machine with the magnetic bearing suspension on the same shaft as the actuator. The result is increased overall efficiency, higher power density and smaller size [2]. 
Active control of a magnetically suspended electrical machine is based on knowledge of the shaft displacement from the center point of the mechanical air gap. In magnetic bearing applications, rotor position measurement has typically been based on eddy current, inductive, or capacitive measurement principles [3]. The cost of non-contact industrial sensor solutions using eddy current sensing technology can, however, amount to $25-30 \%$ of total manufacturing costs for a $60-300 \mathrm{~kW}$ high-speed electrical machine drivetrain. To enable magnetic levitation technology to break through to industry-wide utilization, it is essential that sensor solutions are found that reduce the costs associated with use of such technology in high-speed electrical machines.

Several methods transforming mechanical displacement to an electrical signal using the principle of non-contact measurement have been presented in the literature, including capacitive [4,5], inductive [3], eddy current [6] and optical [7] approaches. The inductive measurement principle has been utilized as a general inductive transducer with a fractional order element [8], as a linear variable differential transformer (LVDT) [9,10], as a variable reluctance differential solenoid transducer (VRDST) [11], as an ironless version in a harsh environment [12], and also as an angular resolver for linear [13] and rotational movements [14]. Differential inductive measurements have been used in LVDT applications to provide high micrometer-scale accuracy even in harsh environments and at industrial scale [15]. A similar inductive measurement approach utilizing one- and twoaxis displacement sensing has been presented for magnetic bearing applications [16,17]. In addition, several sensor signal conditioning methods have been presented using approaches such as envelope detection [16], analog synchronous demodulation [17], fast Fourier transform (FFT) [18], timer-based demodulation [19], frequency counting [9] and integration-based slope conversion $[10,20]$.

The aim of this work is to achieve a robust, compact and cost-efficient rotor displacement sensor design for use in industrial magnetic bearing suspended electrical machine applications, fulfilling the requirements of active control. This paper extends previous research carried out in the field presenting a compact three-axis differential inductive displacement sensor combining all three degrees-of-freedom rotor displacement feedback signals needed in an active magnetic bearing control to the same sensor stator stack. The wound stator part of the sensor utilizes familiar materials and construction well-known to high-speed electrical machine manufacturers, similar to variable reluctance resolvers used for robust rotor angle sensing of electrical machines in harsh environments [14,21]. Sensors are manufactured utilizing the same electrical steel lamination stacks and copper winding materials used in manufacturing of electrical machine stator parts. Well-established manufacturing methods and familiar materials allow cost-efficient manufacturing of the inductive sensor at the same time as the high-speed machine.

A compact design is achieved by integrating the axial axis into the same laminated sensor stator stack as the radial measurement axes as a third measurement axis. The configuration allows axial measurement without decreasing the overall performance of the sensor. In addition, the proposed design and methodology enables cross-coupling between the axes to be reduced to an insignificant level. Moreover, a shorter axial rotor length is achieved compared to the use of an additional set of sensors and laminated stator stacks or conical sensing surfaces on the rotor for axial displacement measurement. A shorter axial length allows better rotordynamic design of the machine and radial detection of axial movement leaves both shaft ends free for application use instead of using one shaft end of the machine as a sensing surface.

Compared to the preceding research, a fully digital signal processing chain utilizing synchronous in-phase and quadrature demodulation is presented. Utilizing modern fully digital signal processing throughout the signal path from excitation to demodulation of the sensor signal helps achieve high bandwidth measurement signals with low noise. The differential nature of the sensor and ratiometric measurement helps to linearize and minimize external disturbances affecting the measurement. The paper is organized as follows. Section 2 presents an analysis of the measurement principle and signal processing methods 
used, the requirement specification derived from the magnetic bearing application, and the electromagnetic sensor design. Verification of the sensor design is carried out by experimental testing in an industrial environment utilizing a $350 \mathrm{~kW}, 15,000 \mathrm{r} / \mathrm{min}$ induction machine with magnetic bearing suspension. The experimental results are described in Section 3. Section 4 concludes the paper.

\section{Three-Axis Inductive Displacement Sensor}

Mechanical displacement of the rotor piece changes the air gap length of the magnetic path formed by one sensor stator pole pair. When using high-permeability electrical steel in the stator and rotor parts, the reluctance of a magnetic circuit is principally defined by the air gap length. Thus, change in air gap length alters the reluctance of the corresponding magnetic circuit, and the reluctance change, in turn, results in a change in the self-inductance of the measurement coil.

By forming an inductive voltage divider branch connecting opposite sensor coils $L_{1}$ and $L_{2}$ in series, the displacement change results in a differential change in coil inductances, which linearizes the measurement. The use of reactive inductive components in the measurement bridge requires that alternating current (ac) excitation be used. The differential change in self-inductance values drives the bridge into unbalance compared to the virtual midpoint of the serially connected resistors $R_{1}$ and $R_{2}$, forming the second branch of the measurement bridge. The voltage difference generated by the unbalance of the bridge can be measured and converted to a corresponding displacement value. The complete measurement principle based on an alternating current (ac) excited four-arm measurement bridge and signal processing chain to convert the ac electrical signal to a value corresponding to the displacement of the rotor piece is illustrated in Figure 1.

The axial measurement utilizes the same measurement principle, but instead of reluctance change based on air gap length change in the radial direction, the reluctance change is achieved by dividing the rotor piece axially to the magnetic and non-magnetic portions. When the rotor piece is centered against the sensor stator, half of the axial sensing pole's tooth width is overlapped with the ferromagnetic rotor electrical steel lamination stack and half of the tooth width is overlapped with non-magnetic material. When there is a change in the rotor position in the axial direction, the portion of magnetic material compared to the non-magnetic material will change on the magnetic sensing path and the self-inductance of the sensing pole pair will change relatively.

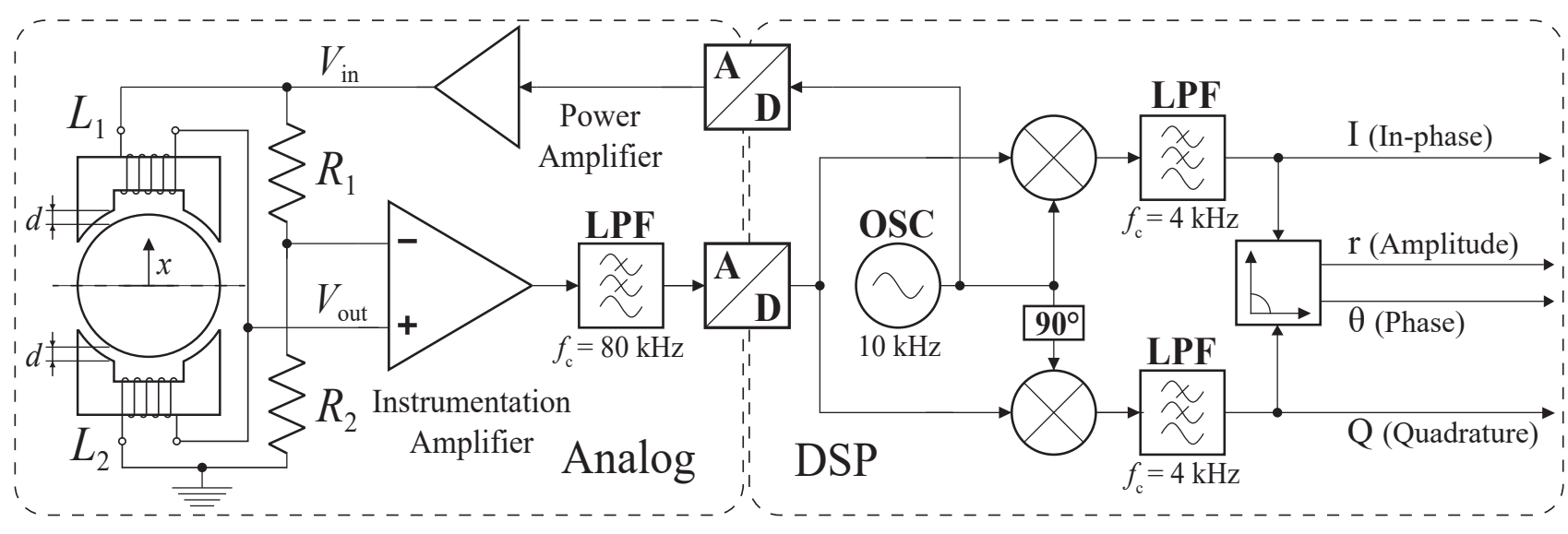

Figure 1. Displacement sensor measurement principle and signal processing chain diagram.

\subsection{Requirement Specification}

The design specification for the sensor solution was derived from the requirements of a conventional industrial magnetic bearing supported electrical machine application and magnetic bearing control theory, and the sensor solution was compared with the specifica- 
tion of alternative displacement measurement technologies. The following specification was set for the sensor design discussed in this paper.

The air gap is defined by the mechanical clearance of the magnetic bearing suspended electrical machine. In the specified application, the air gap was chosen to match the $1 \mathrm{~mm}$ air gap of radial magnetic bearing units of the machine described in [22]. The measurement range of the sensor corresponds to the maximum movement of the rotor limited by the backup bearings. The range of linear measurements of the radial axes is $\pm 300 \mu \mathrm{m}$ and for the axial axis $\pm 600 \mu \mathrm{m}$, which are set by the maximum rotor movement of the machine. Sensor non-linearity is determined by the measurement point deviation from the best straight-line fit to the measured points throughout the measurement range. Non-linearity is set to less than $8 \%$ of the total measurement range in line with the specification of conventional eddy current technology-based displacement sensor linearity [23].

Static displacement measurement of the rotor is required from $0 \mathrm{~Hz}$ to a response up to the $3-10 \mathrm{kHz}$ dynamic region typically found in the magnetic bearing applications and eddy current technology [23]. Within the defined bandwidth, a less than $0.1 \%$ peakto-peak noise level of the full measurement range is desirable. The $0.1 \%$ peak-to-peak noise level value is comparable to over $60 \mathrm{~dB}$ signal-to-noise ratio (SNR) and equals to a 10-bit effective number of bits (ENOB) in analog-to-digital converter (ADC) peak-to-peak resolution. Narrowband noise peaks in the frequency domain are not permissible because they could generate additional audible noise and excite resonance modes in closed-loop operation. The full measurement range of the sensor signal chain is thought to be at least two times the linear measurement range.

The analog domain anti-alias filter and sampling delay will add a phase shift depending on the filter cut-off frequency and sampling rate. Naturally, delays have an effect on the closed-loop control performance and reduce the phase margin. The first-order low-pass anti-alias filter introduces a phase shift of $45^{\circ}$ at the cut-off frequency, and the zero-order hold $(\mathrm{ZOH})$ sampling element introduces a delay of approximately half of a sampling period. The cut-off frequency of the anti-alias filter should be chosen such that suppression at the Nyquist frequency is sufficiently high. For $90 \%$ suppression $(-20 \mathrm{~dB})$ at the Nyquist frequency, the requirement for the sampling rate is twenty times the filter cut-off frequency. With a $5 \mathrm{kHz}$ cut-off frequency linear phase filter operating at a $100 \mathrm{kHz}$ sampling rate, this means an approximately $30 \mu$ sroup delay. All the design requirements set are listed in Table 1.

Table 1. Requirement specification for the inductive sensor.

\begin{tabular}{ll}
\hline \multicolumn{1}{c}{ Parameter } & \multicolumn{1}{c}{ Value } \\
\hline Measurement range (radial/axial) & $\pm 300 \mu \mathrm{m} / \pm 600 \mu \mathrm{m}$ \\
Air gap, $d$ & $1 \mathrm{~mm}$ \\
Non-linearity (deviation from line of best fit) & $<8 \%($ full scale) \\
Frequency response range & $0-3 \mathrm{kHz}(-3 \mathrm{~dB})$ \\
Noise & $<0.1 \%(\mathrm{p}-\mathrm{p}$, full scale) \\
Signal-to-noise ratio (SNR) & $>60 \mathrm{~dB}$ \\
Effective number of bits (ENOB) & $>10 \mathrm{bits}$ \\
Sampling frequency & $>60 \mathrm{kHz}$ \\
& $(-20 \mathrm{~dB}$ suppression at Nyquist frequency) \\
\hline
\end{tabular}

\subsection{Measurement Principle}

The measurement principle of the proposed sensor can be clarified by Figure 1. The relation of the measurement bridge voltage output $V_{\text {out }}$ to the excitation voltage $V_{\text {in }}$ can be defined as the relation of the impedances of $Z_{1}$ and $Z_{2}$ derived from the excitation frequency $\omega$ and differential measurement coil inductances $L_{1}$ and $L_{2}$, which can be resented in the form:

$$
\frac{V_{\text {out }}}{V_{\text {in }}}=\frac{Z_{2}}{Z_{1}+Z_{2}}=\frac{1}{1+\frac{Z_{1}}{Z_{2}}}=\frac{1}{1+\frac{j \omega L_{1}}{j \omega L_{2}}}
$$


Measurement coil inductance $L$ is given as the relation of turn ratio $n$ of the coil and reluctance $\mathcal{R}$ of the magnetic path expressed as:

$$
L=\frac{n^{2}}{\mathcal{R}} .
$$

The windings are assumed to have an equal number of turns. The reluctance of the magnetic path of the measurement coil is determined by the length $l$ of the magnetic path, the effective area $A$ of the magnetic path and the permeability $\mu$ of the material:

$$
\mathcal{R}=\frac{l}{\mu \cdot \mu_{0} \cdot A},
$$

where $\mu_{0}$ is the permeability in a vacuum. The total reluctance of the magnetic path is a sum of the reluctance of the stator part $\mathcal{R}_{\mathrm{s}}$, reluctance of the rotor part $\mathcal{R}_{\mathrm{r}}$ and reluctance of the air gap $\mathcal{R}_{\mathrm{a}}$ :

$$
\mathcal{R}_{\text {tot }}=\mathcal{R}_{\mathrm{s}}+\mathcal{R}_{\mathrm{r}}+\mathcal{R}_{\mathrm{a}},
$$

where stator and rotor parts are, in general, frequency dependent due to the eddy currents. The methodology on how to take this into account is described by [24]. The presented above equation can be simplified by defining the rotor and stator part reluctance as a constant $\mathcal{R}_{0}$ and separating the rest into variable gap length $d$ and constant coefficient $k$ :

$$
\begin{gathered}
\mathcal{R}_{\text {tot }}=\mathcal{R}_{0}+\frac{2 d}{\mu \cdot \mu_{0} \cdot A}=\mathcal{R}_{0}+d \cdot k, \\
k=\frac{2}{\mu_{0} \cdot \mu \cdot A} .
\end{gathered}
$$

The opposite positioned measurement coil inductances $L_{1}$ and $L_{2}$ can then be written based on the mechanical displacement $x$ as:

$$
\begin{aligned}
& L_{1}=n^{2} /\left(\mathcal{R}_{0}+k(d-x)\right), \\
& L_{2}=n^{2} /\left(\mathcal{R}_{0}+k(d+x)\right) .
\end{aligned}
$$

Substituting the inductance equations into (1) gives:

$$
\frac{V_{\text {out }}}{V_{\text {in }}}=\frac{1}{1+\frac{j \omega L_{1}}{j \omega L_{2}}}=1 /\left(1+\frac{n^{2} /\left(\mathcal{R}_{0}+k(d-x)\right)}{n^{2} /\left(\mathcal{R}_{0}+k(d+x)\right)}\right) .
$$

With algebraic operations, the expression is simplified as follows:

$$
\frac{V_{\text {out }}}{V_{\text {in }}}=\frac{1}{2}-\frac{k \cdot x}{2 \cdot\left(\mathcal{R}_{0}+k \cdot d\right)} .
$$

The result is a linear equation of the form:

$$
\frac{V_{\text {out }}}{V_{\text {in }}}=\frac{1}{2}-K \cdot x,
$$

which verifies that the differential setup of the measurement coils gives linear output with the sensitivity:

$$
K=\frac{k}{2 \cdot\left(\mathcal{R}_{0}+k \cdot d\right)} .
$$


However, the simplified analysis is based on an assumption of ideal windings without any resistive losses in the windings. The analysis can be expanded by adding the series resistance $R_{\mathrm{s}}$ of the coil to the Equation (1) expressed as:

$$
\frac{V_{\text {out }}}{V_{\text {in }}}=\frac{1}{1+\frac{Z_{1}}{Z_{2}}}=\frac{1}{1+\frac{R_{s}+j \omega L_{1}}{R_{s}+j \omega L_{2}}} .
$$

The effect of stray resistance on the linearity of the sensor and measurement error is shown in Figure 2. The resistance of the coils is assumed to be equal.
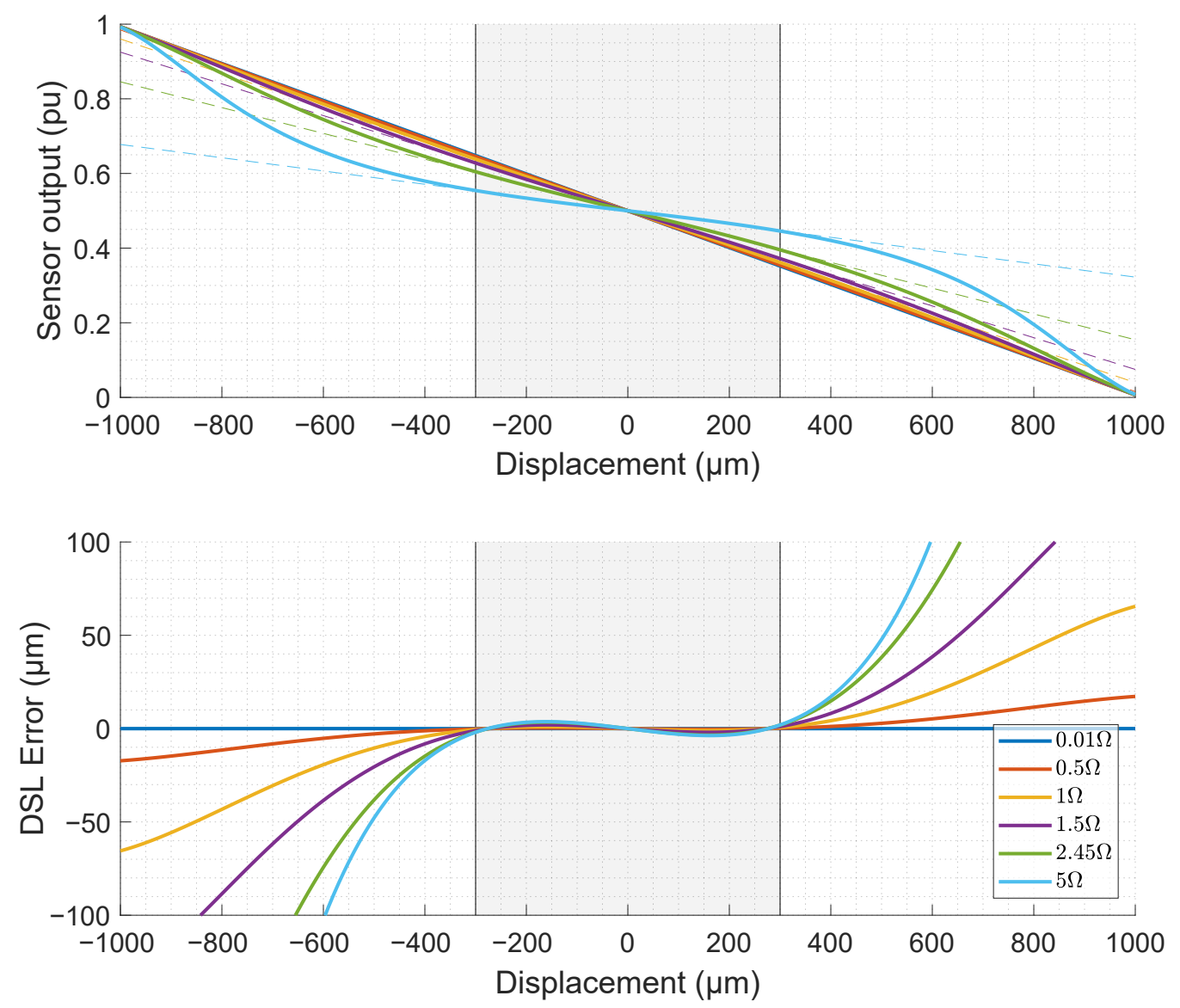

Figure 2. Effect of measurement coil stray series resistance on the linearity of the inductive measurement bridge output based on an analytical model of the electromagnetic circuit. Output error (lower figure) calculated as deviation from the best straight-line fit (dashed line) over the measurement range (grey area).

\subsection{Electromagnetic Design}

The main dimensions of the sensor, presented in Figure 3, are defined by the dimensions of the electrical machine. The rotor diameter defines the minimum inner diameter, and the outer diameter is defined by the usable radial space inside the machine frame structure. Axial length was chosen based on the space requirement of the sensor winding such that the sensor is still easily manufacturable. Air gap length depends on the actual mechanical clearance of the rotor system. An additional safety margin was added, to account for manufacturing tolerances and to allow full rotor movement along the mechanical safety bearings without any risk of touching the sensor mechanically.

Lamination stack material selection was carried out so as to choose a material with low eddy current and hysteresis losses considering the $10 \mathrm{kHz}$ excitation frequency and the material chosen was a compromise between electromagnetic properties and use of material common throughout the electrical machine manufacturing industry. The magnetic flux 
density was designed to utilize only a small portion of the linear region of the $\mathrm{BH}$ curve and flux density was restricted by the power handling capability of the excitation amplifier. The winding turn number should be adjusted to fit the properties of the exciting power amplifier's voltage level and current driving capability. Electromagnetic design parameters are given in Table 2 and the design FEM analysis in Figure 4.
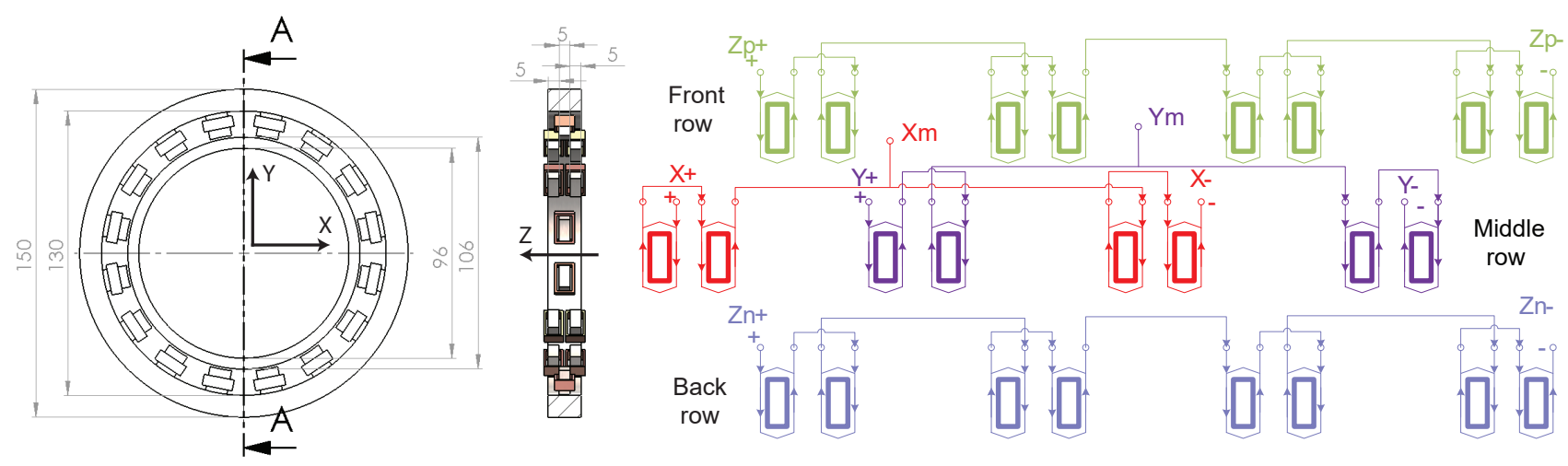

Figure 3. Mechanical dimensions of the displacement sensor and the winding scheme for all three measurement axes.

The axial measurement is achieved using the same stator stack geometry as for radial direction measurement, but all the four pole pairs of the stack are connected in series, as illustrated in the connection diagram in the Figure 3. All the four pole pairs of the stator stack are used to reject the sensitivity of the axial sensing circuit to the radial displacement change. The differential measurement for the axial direction is achieved by utilizing two sensor identical stacks in axial direction, defined as front and backrow in Figure 3. The rotor piece width in the example sensor design is $10 \mathrm{~mm}$, from which results that in the centered position the rotor piece will overlap with the axial tooth only by half of the tooth width. This overlapping on both halves of the axial sensor stack as illustrated in Figure 5 allows the self-inductance of the front and back row sensing pole pairs change differentially when the rotor position changes in the axial direction.

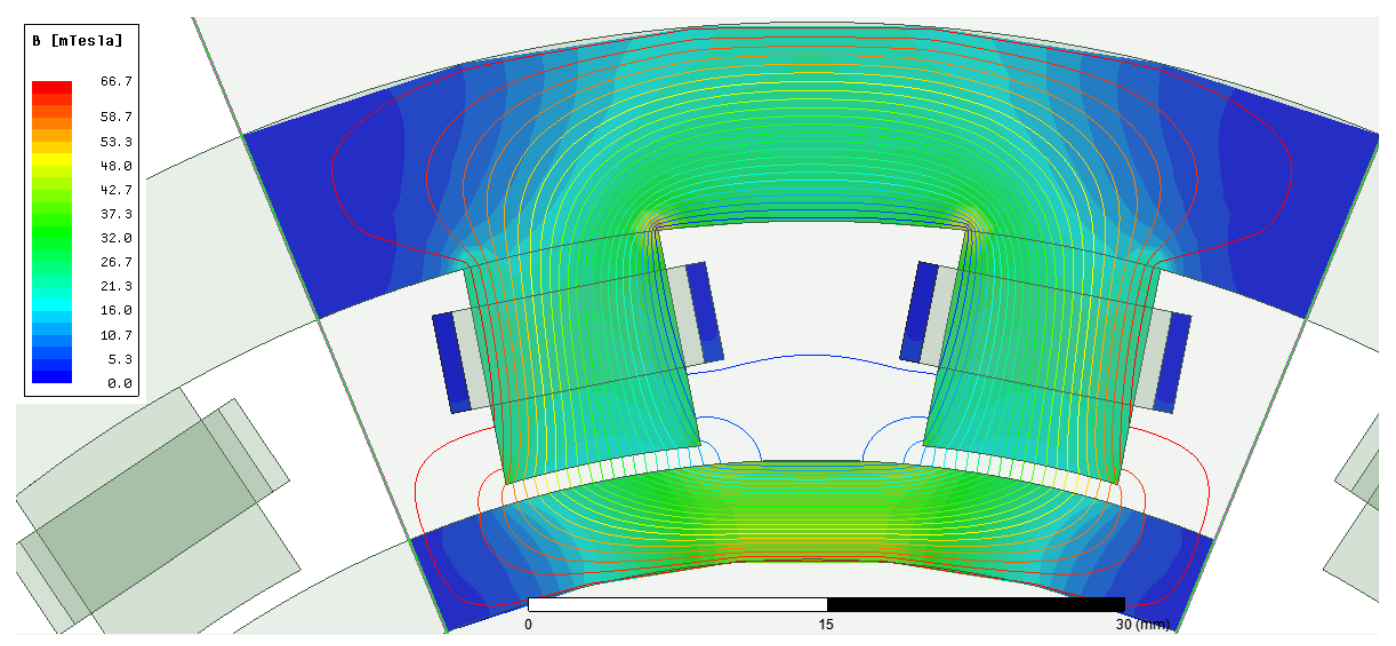

Figure 4. FEM analysis of the sensor. 

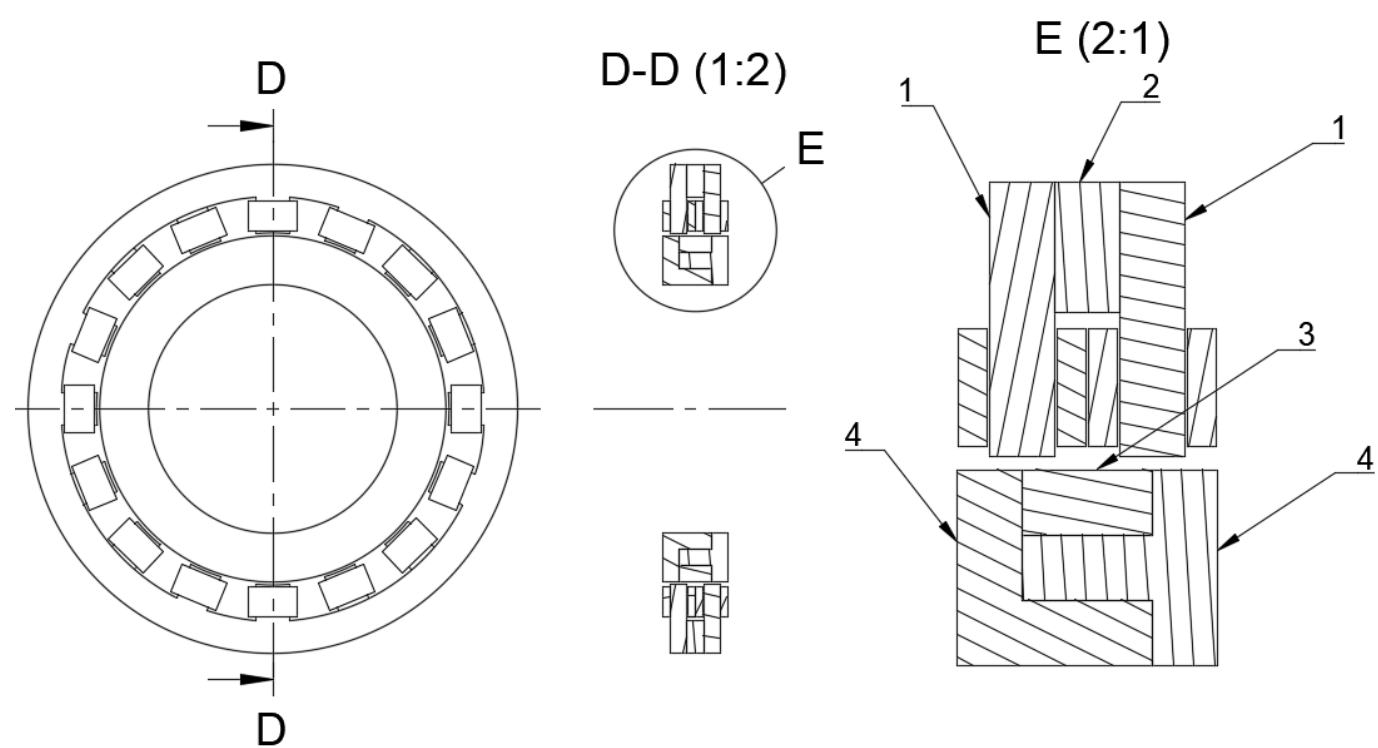

Figure 5. Cross-section of the sensor at poles corresponding to axial measurements. Numbers denote the following parts: 1-axial sensor stator stack, 2-radial sensor stator stack, 4-non-magnetic aluminium holding parts, 3-laminated rotor part.

Table 2. Design parameters.

\begin{tabular}{ll}
\hline \multicolumn{1}{c}{ Parameter } & \multicolumn{1}{c}{ Value } \\
\hline Number of turns per pole, $n$ (radial/axial) & $35 / 25$ \\
Inductance per pole pair, $L$ (radial/axial) & $208 \mu \mathrm{H} / 53 \mu \mathrm{H}$ \\
Excitation voltage & $10.3 \mathrm{~V}$ \\
Excitation frequency & $10 \mathrm{kHz}$ \\
Stator/rotor sheet material & SURA M270-35A $(0.35 \mathrm{~mm})$ \\
\hline
\end{tabular}

\subsection{Phase Sensitive Signal Processing}

Displacement sensor operation is based on measuring the voltage signal output from all three axes. The measurement coils are driven by the same amplified excitation signal common to all the axes. The output of the sensor is an alternating current signal at the frequency of the excitation signal, which needs to be demodulated to obtain a signal corresponding to the static mechanical displacement.

The demodulation can be achieved using simple amplitude-based demodulation techniques such as a peak detector or root-mean-square (rms) detector circuit, or by rectifying and filtering the output signal. The drawback of these demodulation methods is that they can only observe the magnitude change of the measured signal, and phase information is lost. By synchronizing the excitation signal generation and measurement, it is possible to use phase-sensitive detection methods. Use of a synchronous demodulation scheme using in-phase and quadrature signals preserve amplitude and phase information without losing information from the measurement signal. Preserving the phase information allows delay and phase shift in the system to be taken into account and compensated during the signal processing. Synchronous demodulation acting as a narrow bandpass filter at the excitation frequency helps to achieve a high dynamic range even if off-band or spurious noise is present in the measurement system [25]. These properties of phase-sensitive demodulation schemes allow a low-noise high-bandwidth measurement signal to be obtained in magnetic bearing control systems, where the displacement sensor typically needs to be positioned next to a pulse-width modulated magnetic bearing power circuit coupling, which introduces noise into the sensor circuit.

Excitation signal generation and synchronous demodulation with a phase lock can be constructed using a fully digital principle with the addition of A/D- and D/A-converters. 
In a magnetic bearing application, a shared digital signal processing system can be utilized with the closed-loop position control. The fully digital signal processing principle is presented in Figure 1. The sine wave excitation signal is generated in the digital domain and fed through a digital-to-analog converter to the analog power amplifier. The measurement bridge output voltages from all three axes are brought back to the digital domain through an analog-to-digital converter. The amplified excitation signal is also measured back to the digital domain to achieve ratio-metric measurement that compensates possible gain and phase drift in the analog measurement circuit. The excitation signal $V_{\text {exc }}(t)$ is digitally generated with $0^{\circ}$ and $90^{\circ}$ phase shifts and can be expressed in a complex plane as

$$
V_{\text {exc }}(t)=\sqrt{2} e^{-i \omega t}=\sqrt{2} \cos (\omega t)-i \sqrt{2} \sin (\omega t),
$$

where $\omega$ corresponds the angular frequency of the excitation signal. Similarly the measurement signal $V_{\mathrm{m}}(t)$ can be defined in the complex plane as:

$$
\begin{aligned}
V_{\mathrm{m}}(t) & =\sqrt{2} r \cos (\omega t+\theta) \\
& =\frac{r}{\sqrt{2}}\left(e^{i(\omega t+\theta)}+e^{-i(\omega t+\theta)}\right),
\end{aligned}
$$

where $r$ corresponds to the measurement signal amplitude and $\theta$ phase shift. Mixing the measurement signal with the generated local oscillator excitation signal down-converts the measurement signal in the frequency domain to the dc level but also generates double the excitation frequency component defined in the complex plane as:

$$
\begin{aligned}
Z(t) & =X(t)+i Y(t)=V_{\mathrm{m}}(t) \cdot V_{\mathrm{exc}}(t) \\
& =r\left(e^{i((\omega-\omega) t+\theta)}+e^{-i((\omega+\omega) t+\theta)}\right) .
\end{aligned}
$$

The double frequency $(2 \omega)$ signal can be low-pass filtered or averaged (integrated) over the period. The resulting filtered signal corresponds to demodulated in-phase and quadrature signals of the displacement measurement. The dynamic bandwidth of the measured displacement signal can be defined and limited by a digital domain low-pass filter located after the mixing process.

After the demodulation process, two complex form values for both the measurement signal and the excitation signal are obtained. Dividing the measurement signal by the excitation signal in a complex plane gives a ratiometric result whose phase is aligned to the phase of the measured excitation signal. The operation principle compensates for the effect of the total signal chain delay from the excitation output to the measurement input. Thus, the ratiometric measurement result can be declared as the real part of the complex value, with the imaginary part being insignificantly small.

\section{Experimental Results}

The designed sensor was manufactured to be able to verify the sensor design and its operational performance in a real industrial application. The wound sensor stator stack and laminated piece on the rotor shaft of the electrical machine are presented in Figure 6. The sensor was calibrated on a table test rig utilizing a linear XYZ-axis microscrew stage. After calibration, the sensor was installed in a $350 \mathrm{~kW}, 15,000 \mathrm{r} / \mathrm{min}$ industrial solid-rotor induction motor. Two sensors were installed and functioned as part of the magnetic bearing control feedback loop, while providing radial and axial displacement measurements of the machine's rotor from both ends of the machine. The laboratory test setup, including the high-speed induction machine, is shown in Figure 7.

The motor was driven by an ABB ACS800 industrial variable frequency drive. In the practical tests, the rotational speed was limited to $1200 \mathrm{r} / \mathrm{min}$ for safety reasons. Magnetic bearing position control and sensor signal digital processing were implemented in a Beckhoff TwinCAT real-time environment utilizing a Beckhoff Industrial PC, EtherCAT 
connected power sources driving the magnetic bearing coils and Beckhoff analog I/O terminals handling the interface to the analog circuitry and sensor coils.

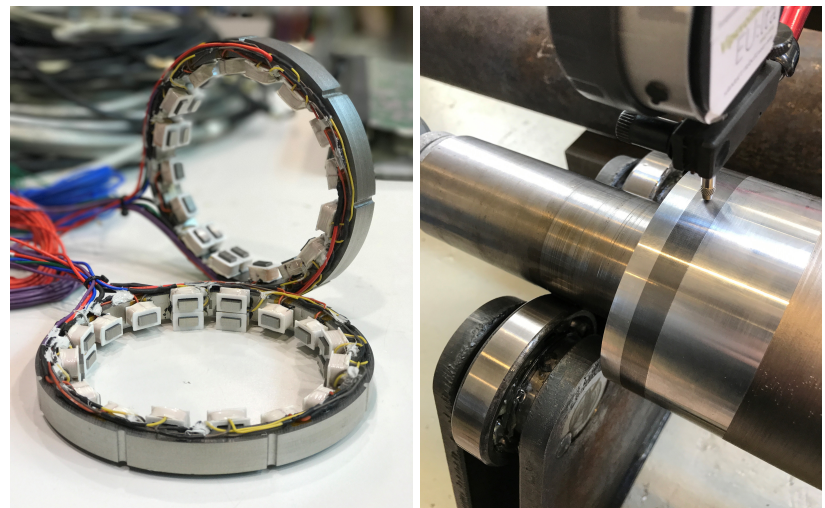

Figure 6. Inductive differential three-axis rotor displacement sensor. Laminated stator stacks with windings (left) and laminated rotor sensing surface with non-magnetic aluminium surroundings installed on electrical machine rotor (right).

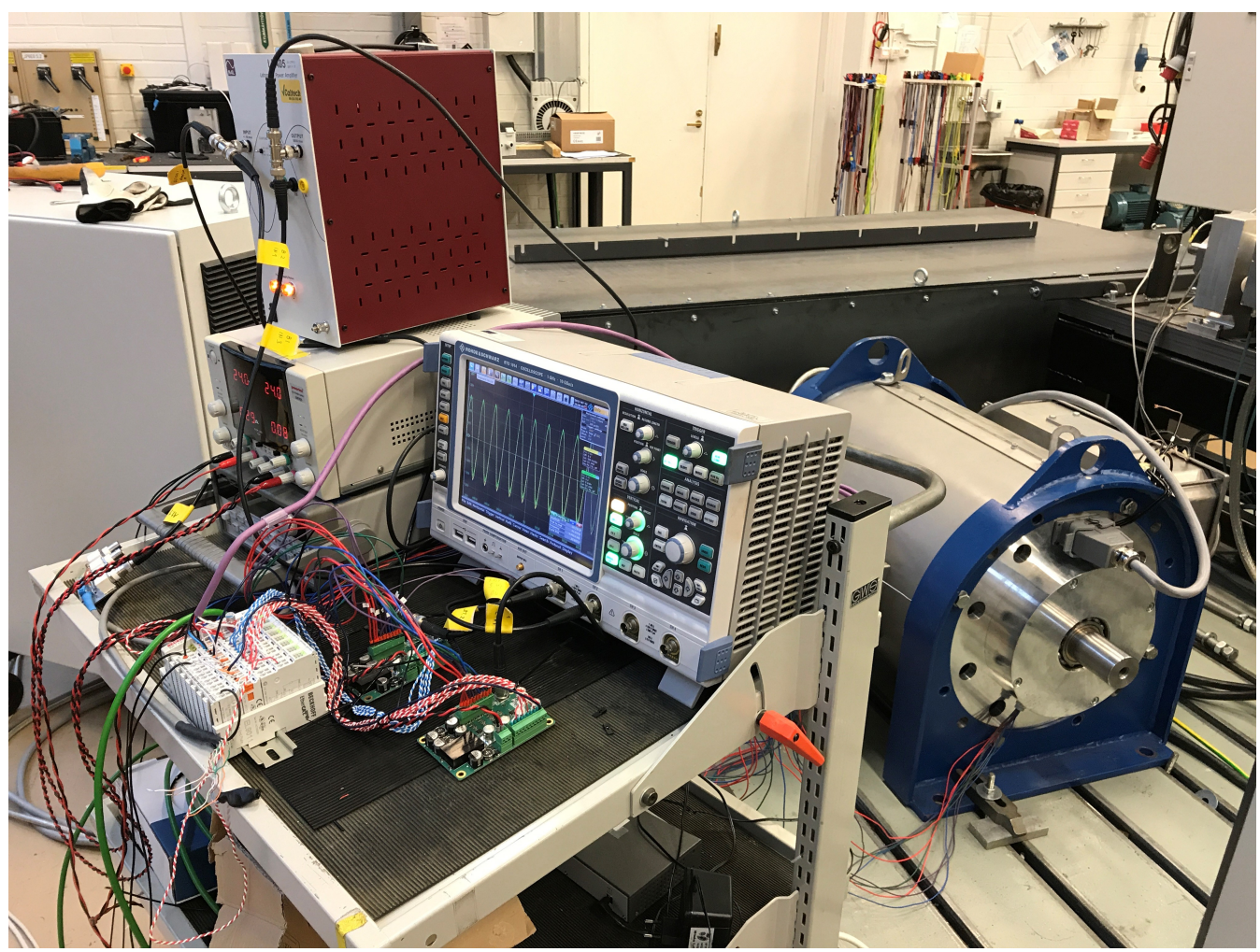

Figure 7. Measurement setup with a $350 \mathrm{~kW}, 15,000 \mathrm{r} / \mathrm{min}$ industrial high-speed induction machine and interface electronics for the displacement sensor.

A digitally generated $10 \mathrm{kHz}$ sine wave excitation signal was driven through the Beckhoff EL4732 $\pm 10 \mathrm{~V}, 16$-bit, 100,000 samples per second analog output terminal acting as a digital-to-analog converter. The N4L LPA05A laboratory power amplifier amplified the excitation signal to 10.7 Vrms driving all the parallel-connected measurement coils. The excitation signal and measurement signals were converted back to the digital domain through instrumentation amplifiers and by utilizing the Beckhoff EL3702 $\pm 10 \mathrm{~V}$, 16-bit, 100,000 samples per second EtherCAT analog input terminal as an analog-to-digital converter. The demodulation, filtering and ratiometric signal processing of the digital measurement signals were implemented in the Beckhoff TwinCAT environment. The dynamic properties of the displacement measurement were determined by the dynamics 
of the low-pass filtering after the frequency mixer. The low-pass filter gain and phase frequency response are presented in Figure 8.
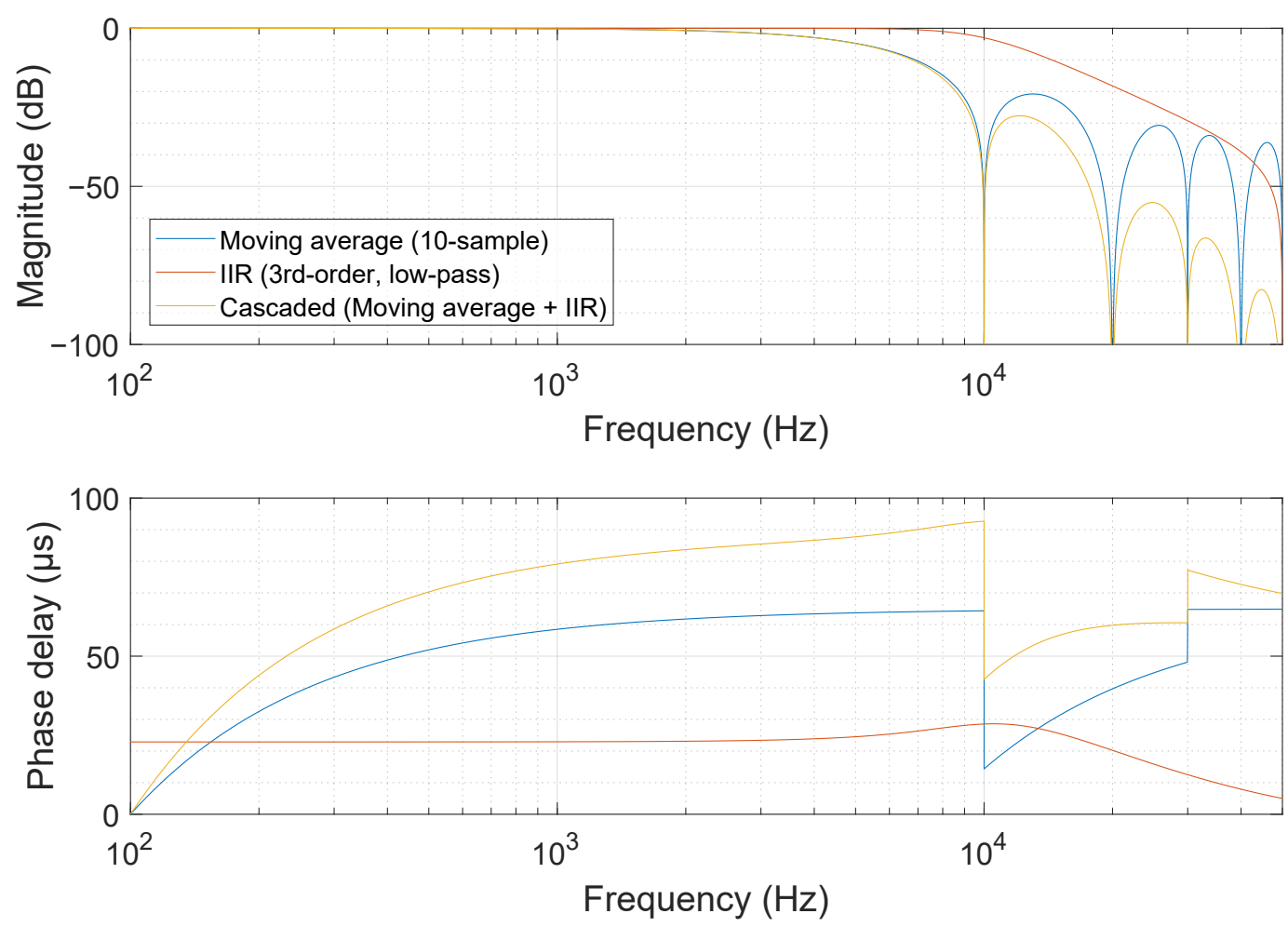

Figure 8. Magnitude response and phase delay of the digital low-pass filters filtering the measurement signal during demodulation. Filtering consists of a 10-sample moving average filter combined with a 3rd-order low-pass IIR filter operated at $100 \mathrm{kHz}$ sampling frequency. The filtering results in a $4 \mathrm{kHz}-3 \mathrm{~dB}$ cut-off frequency with $87 \mu$ s phase delay at the cut-off frequency.

\subsection{Static Accuracy and Linearity}

The sensor was calibrated and measured against the OptoSigma precision XYZ axis linear stage with manual micrometer screws. A first-order linear fit was used with the measured points over the specified measurement range of $\pm 300 \mu \mathrm{m}$ radial and $\pm 600 \mathrm{um}$ axial axes. After the linear fit, measurement point deviation from the fitted straight line was calculated. The static measurement results after calibration from all three axes are presented in Figure 9. Note that the static cross-coupling effects between the three axes were noticed not to be separable from the mechanical inaccuracy of the test setup, including linear stage and rotor piece machining inaccuracies. Measurable cross-coupling between axial and radial axes over the measurement range was less than $2 \mu \mathrm{m}$. Deviation from straight-line fit was smaller than $2 \%$ in the specified $\pm 300 \mu \mathrm{m}$ measurement range for the radial direction. Deviation from the straight-line fit was smaller than $0.5 \%$ in the specified $\pm 600 \mu \mathrm{m}$ measurement range for the axial direction. The better linearity of the axial direction is a result of a longer total mechanical range with respect to the size of the measurement range compared to the radial axes. The maximum measurement range for radial direction is $\pm 1000 \mu \mathrm{m}$ and for axial direction $\pm 2500 \mu \mathrm{m}$. Experimentally measured non-linearity values were significantly less than the $8 \%$ requirement specified for non-linearity in Section 2.

\subsection{Dynamic Performance}

Sensor performance was tested while the sensor was part of the feedback loop of the active magnetic bearing control system. First, the noise level was measured at standstill with the rotor lying on the safety bearings. Noise level measurement from the radial and 
axial axes is presented in Figure 10. Noise of $0.11 \mu \mathrm{m}$ rms was found in the radial axes and $0.35 \mu \mathrm{m}$ in the axial axis. These values correspond to $0.7 \mu \mathrm{m}$ and $2.1 \mu \mathrm{m}$ peak-to-peak noise, respectively. The noise levels scaled relative to maximum measurement range of $\pm 1000 \mu \mathrm{m}$ for radial and $\pm 2500 \mu \mathrm{m}$ for axial direction results as $-76 \mathrm{~dB}$ for radial direction and $-74 \mathrm{~dB}$ for axial direction measurement. From the frequency domain analysis, it was seen that the noise is evenly distributed over the measurement bandwidth and no narrowband noise peaks are visible.
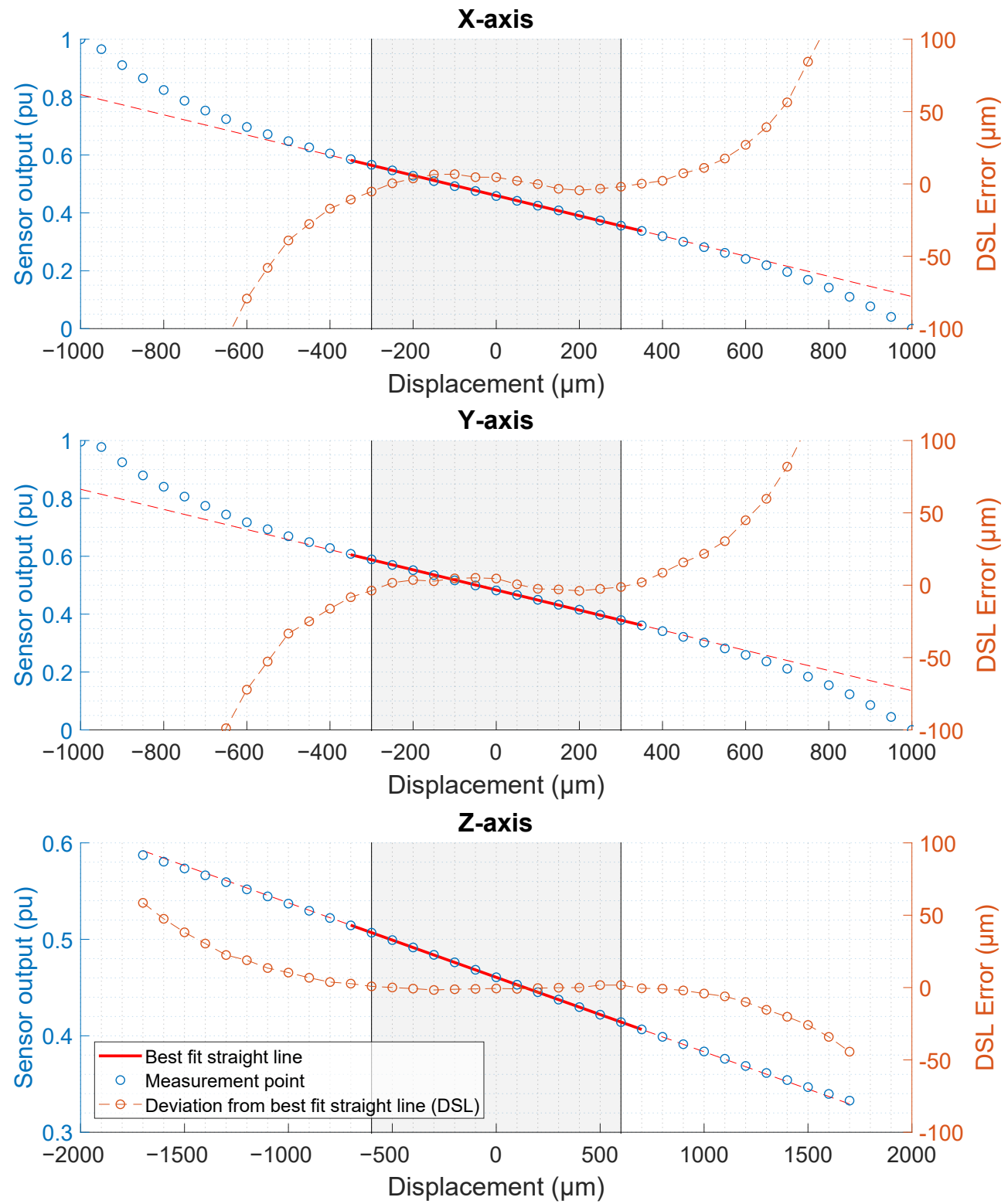

Figure 9. Static sensor linearity measurement on a manual three-axis linear stage. Measurement points (blue) and deviation points (orange) from the best straight-line fit (red). The grey area corresponds to the specified linear measurement range. The best straight-line fit corresponds to a radial gain of $-2870 \mu \mathrm{m} / \mathrm{pu}$ and axial gain of $-12,950 \mu \mathrm{m} / \mathrm{pu}$. The maximum deviation from the best straight-line fit (DSL) throughout the linear measurement range is $7 \mu \mathrm{m}$ for radial axes and $2 \mu \mathrm{m}$ for the axial axis.

After noise measurement, and to verify proper operation in a noisy industrial environment, the dynamic performance was tested while the rotor was rotating at a moderate velocity of $1200 \mathrm{r} / \mathrm{min}$ and levitated by the active magnetic bearing control. It should 
be noted that the controller used in the experimental tests included additional control functions. Two types of measured displacement signals were recorded while levitating and rotating the machine. The higher amplitude signal visible in Figure 11 presents the actually measured rotor displacement by the sensor after the demodulation and low-pass filtering. The lower amplitude signal in Figure 12 presents the signal driven to the magnetic bearing control input with additional signal processing [26]. The harmonic content corresponds mainly to unbalance, runout and other mechanical non-idealities present in the system, and the harmonics generate unnecessary control action if not removed from the controller input. The first, second, third, fourth and fifth harmonics of the rotational frequency were removed from the radial position controller input signal, and correspondingly the first, second and fourth harmonics were removed from the axial controller input.
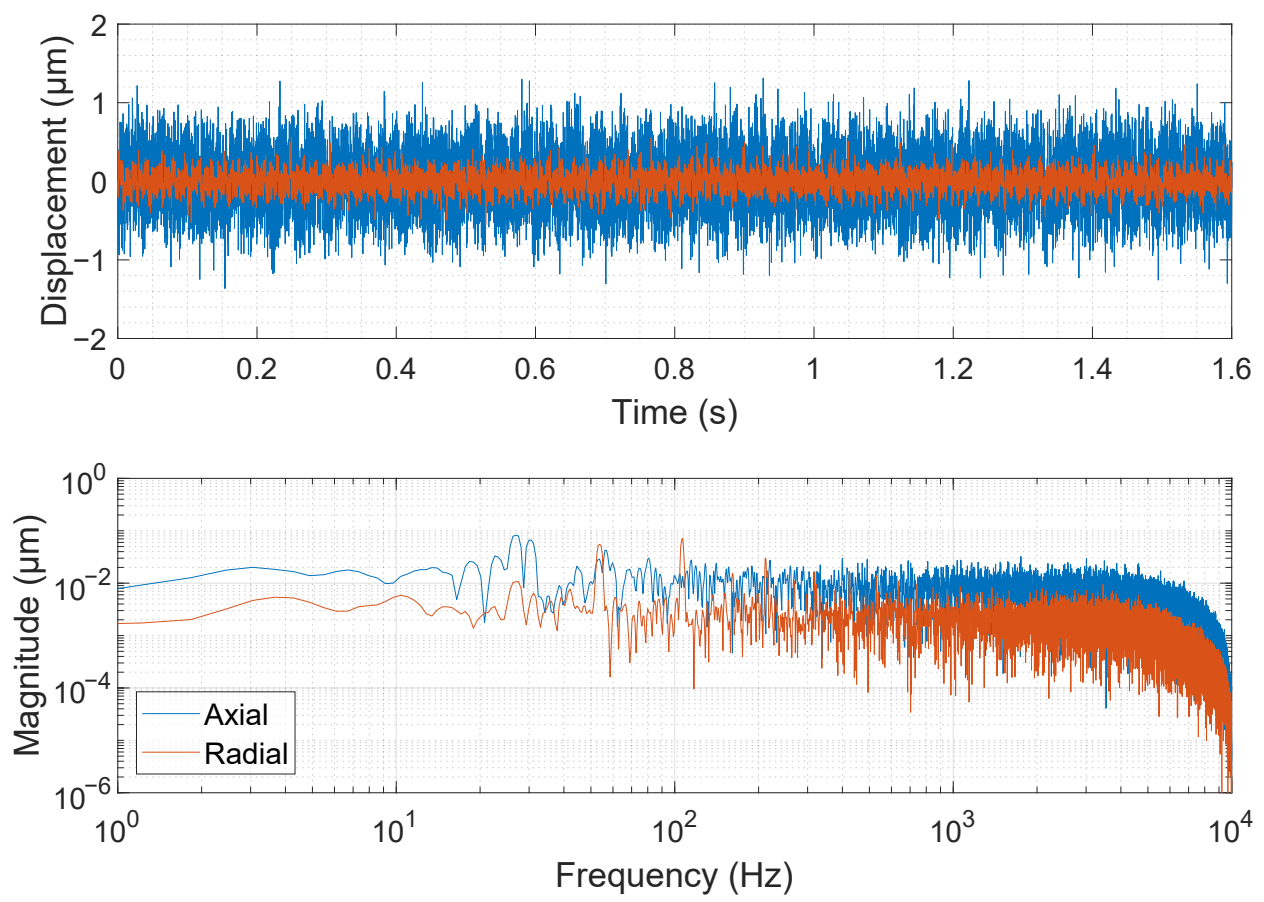

Figure 10. Measured noise level when the machine's rotor is lying statically on the safety bearings. Frequency domain fast Fourier transform (FFT) consists of 32,768 samples at $20 \mathrm{kHz}$ sampling frequency.
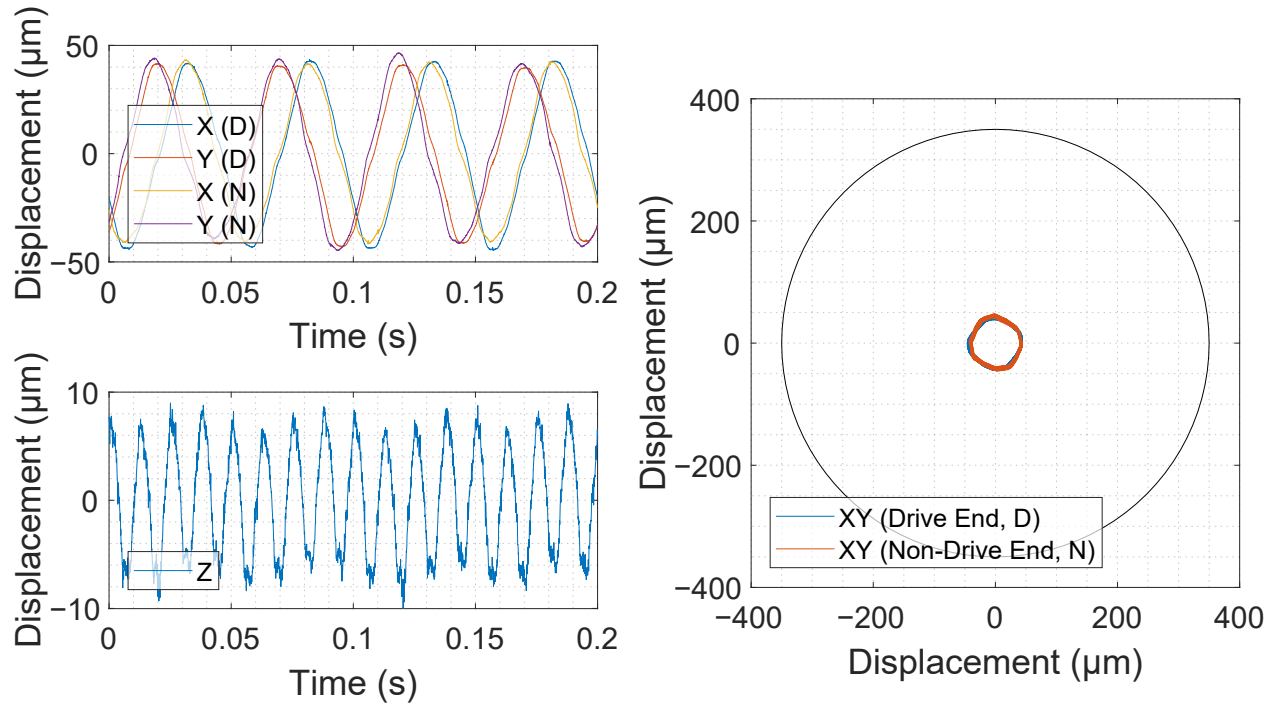

Figure 11. Rotor displacement measurement signals from two inductive displacement sensors placed at each end of the machine while the rotor is levitated and rotating at $1200 \mathrm{r} / \mathrm{min}$. 

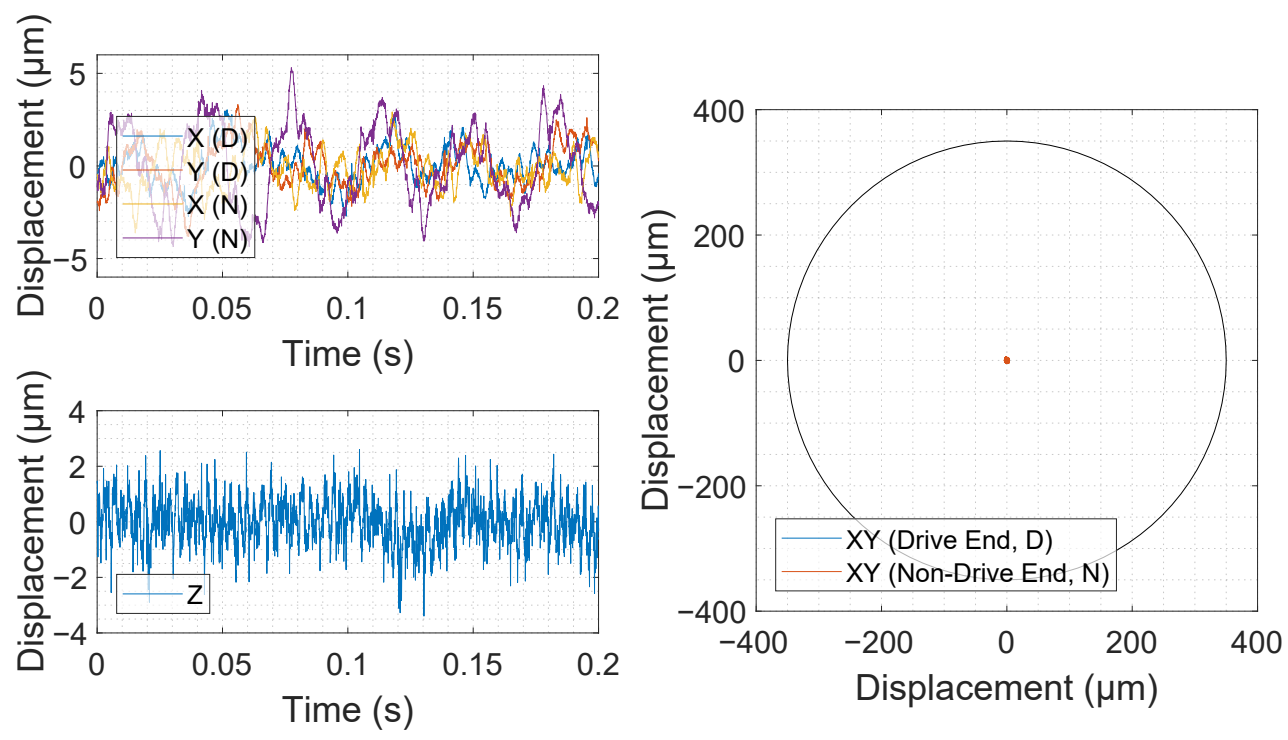

Figure 12. Rotor displacement measurement signals fed to the rotor position controller of the magnetic bearing system from two inductive displacement sensors while the rotor is levitated and rotating at $1200 \mathrm{r} / \mathrm{min}$. Note: The difference from the measurement signals presented in Figure 11 is the removal from the controller input of harmonic content synchronous to the rotational frequency.

\section{Conclusions}

This paper presented a three-dimensional position sensor design and phase-sensitive digital signal processing method. The proposed sensor design was validated specifically for use in active magnetic bearing suspended electrical machines, but can be used widely whenever three-axis non-contact displacement measurement is required and a ferromagnetic target piece can be used. An analytical method was used to estimate the sensor's non-linearity based on the real losses of the excitation and measurement circuit. The displacement sensor design and digital signal processing scheme were verified by experimental tests to ascertain whether they met the requirements of magnetic bearing applications. It was found that the design and scheme operated appropriately in closed-loop operation in an industrial electrical drive system. The custom sensor design and signal processing scheme provide an alternative solution to commercial eddy current-based sensors and, based on specification values, exhibit comparable performances. No cross-coupling or interference in the frequency domain was detected between the measured sensor signal and 400 volts pulse-width-modulation (PWM) driven motor coils of the industrial induction machine when rotating $1200 \mathrm{r} / \mathrm{min}$ at no load. The dynamic performance of the sensor was limited in the experimental setup by the bandwidth of the analog measurement device. Custom-made signal condition electronics would allow an increase in the sampling rate and excitation frequency, providing additional bandwidth and less delay by allowing higher cut-off frequency filters to be used. However, the effect of the higher excitation frequency on the overall performance of the sensor should be studied as a part of future research work. Other disadvantages of the mentioned sensor technology include the requirement for a laminated electrical steel target piece structure, additional sensor electronics when interfacing with external measurement systems and a number of sensitive electrical wires between the electronics and sensor installed inside the electrical machine.

Author Contributions: T.S.: conceptualization, software, validation, investigation, writing-original draft, and visualization. A.S.: conceptualization, methodology, formal analysis, investigation, writing - original draft, visualization, and funding acquisition. P.J.: conceptualization and resources. J.V.: conceptualization and resources. N.N.: writing—original draft and writing-review and editing. R.J.: writing-review and editing and supervision. O.P.: writing-review and editing, supervision, and project administration. All authors have read and agreed to the published version of the manuscript. 
Funding: This research was funded by European Regional Development Fund (ERDF) (grant number A71875).

Institutional Review Board Statement: Not applicable.

Informed Consent Statement: Not applicable.

Conflicts of Interest: The authors declare no conflict of interest. The funders had no role in the design of the study; in the collection, analyses, or interpretation of data; in the writing of the manuscript, or in the decision to publish the results.

\section{References}

1. Soni, T.; Dutt, J.K.; Das, A.S. Parametric Stability Analysis of Active Magnetic Bearing Supported Rotor System with a Novel Control Law Subject to Periodic Base Motion. IEEE Trans. Ind. Electron. 2020, 67, 1160-1170. [CrossRef]

2. Schweitzer, G.; Maslen, E.H. Magnetic Bearings-Theory, Design and Application to Rotating Machinery; Springer: Berlin, Germany, 2009.

3. George, B.; Tan, Z.; Nihtianov, S. Advances in Capacitive, Eddy Current, and Magnetic Displacement Sensors and Corresponding Interfaces. IEEE Trans. Ind. Electron. 2017, 64, 9595-9607. [CrossRef]

4. Jastrzebski, R.P.; Matusiak, D.; Sillanpää, T.; Romanenko, A.; Jaatinen, P.; Tolsa, K.; Lindh, T.; Pyrhönen, O. Modelling and evaluation of radial-axial PCB capacitive position sensor prototype. In Proceedings of the 2014 16th European Conference on Power Electronics and Applications, Lappeenranta, Finland, 26-28 August 2014; pp. 1-8. [CrossRef]

5. Jeon, S.; Ahn, H.J.; Han, D.C.; Chang, I.B. New design of cylindrical capacitive sensor for on-line precision control of AMB spindle. IEEE Trans. Instrum. Meas. 2001, 50, 757-763. [CrossRef]

6. Wang, K.; Zhang, L.; Han, B.; Chen, S. Analysis and Experiment of Self-Differential Eddy Current Displacement Sensor for AMBs Used in Molecular Pump. IEEE Trans. Instrum. Meas. 2018, 1-10. [CrossRef]

7. Lin, C. Techniques for the measurement of air-bearing separation-A review. IEEE Trans. Magn. 1973, 9, 673-677. [CrossRef]

8. Partha Sarathi, V.; Uma, G.; Umapathy, M. Realization of Fractional Order Inductive Transducer. IEEE Sens. J. 2018, $18,8803-8811$. [CrossRef]

9. Gunasekaran, V.; George, B.; Aniruddhan, S.; Janardhanan, D.D.; Palur, R.V. Performance Analysis of Oscillator-Based Read-Out Circuit for LVDT. IEEE Trans. Instrum. Meas. 2019, 68, 1080-1088. [CrossRef]

10. Ganesan, H.; George, B.; Aniruddhan, S.; Haneefa, S. A Dual Slope LVDT-to-Digital Converter. IEEE Sens. J. 2019, 19, 868-876. [CrossRef]

11. Reinholz, B.A.; Seethaler, R.J. Design and Validation of a Variable Reluctance Differential Solenoid Transducer. IEEE Sens. J. 2019, 19, 11063-11071. [CrossRef]

12. Grima, A.; Di Castro, M.; Masi, A.; Sammut, N. Thermal Study of the Ironless Inductive Position Sensors Installed on the LHC Collimators. IEEE Trans. Nucl. Sci. 2019, 66, 688-695. [CrossRef]

13. Sun, L.; Taylor, J.; Guo, X.; Cheng, M.; Emadi, A. A Linear Position Measurement Scheme for Long-Distance and High-Speed Applications. IEEE Trans. Ind. Electron. 2020, 68, 4435-4447. [CrossRef]

14. Sun, L.; Taylor, J.; Callegaro, A.D.; Emadi, A. Stator-PM-Based Variable Reluctance Resolver with Advantage of Motional Back-EMF. IEEE Trans. Ind. Electron. 2020, 67, 9790-9801. [CrossRef]

15. Masi, A.; Brielmann, A.; Losito, R.; Martino, M. LVDT Conditioning on the LHC Collimators. IEEE Trans. Nucl. Sci. 2008, 55, 67-75. [CrossRef]

16. Wang, K.; Zhang, L.; Le, Y.; Zheng, S.; Han, B.; Jiang, Y. Optimized Differential Self-Inductance Displacement Sensor for Magnetic Bearings: Design, Analysis and Experiment. IEEE Sens. J. 2017, 17, 4378-4387. [CrossRef]

17. Chen, S.C.; Le, D.K.; Nguyen, V.S. Inductive Displacement Sensors with a Notch Filter for an Active Magnetic Bearing System. Sensors 2014, 14, 12640-12657. [CrossRef]

18. Flammini, A.; Marioli, D.; Sisinni, E.; Taroni, A. A multichannel DSP-based instrument for displacement measurement using differential variable reluctance transducer. IEEE Trans. Instrum. Meas. 2005, 54, 178-183. [CrossRef]

19. Reverter, F.; Gasulla, M. Timer-Based Demodulator for AM Sensor Signals Applied to an Inductive Displacement Sensor. IEEE Trans. Instrum. Meas. 2017, 66, 2780-2788. [CrossRef]

20. Philip, N.; George, B. Design and Analysis of a Dual-Slope Inductance-to-Digital Converter for Differential Reluctance Sensors. IEEE Trans. Instrum. Meas. 2014, 63, 1364-1371. [CrossRef]

21. Xiao, L.; Li, Z.; Bi, C. An Optimization Approach to Variable Reluctance Resolver. IEEE Trans. Magn. 2020, 56, 1-5. [CrossRef]

22. Smirnov, A.; Uzhegov, N.; Sillanpää, T.; Pyrhönen, J.; Pyrhönen, O. High-Speed Electrical Machine with Active Magnetic Bearing System Optimization. IEEE Trans. Ind. Electron. 2017, 64, 9876-9885. [CrossRef]

23. Data Sheet, Bently Nevada Asset Condition Monitoring, General Electric Company. In Bently Nevada 3300 XL NSv Proximity Transducer System; Bently Nevada: Minden, NV, USA, 2011.

24. Meeker, D.; Maslen, E.; Noh, M. An augmented circuit model for magnetic bearings including eddy currents, fringing, and leakage. IEEE Trans. Magn. 1996, 32, 3219-3227. [CrossRef] 
25. Gervasoni, G.; Carminati, M.; Ferrari, G. Lock-In Amplifier Architectures for Sub-ppm Resolution Measurements. In Advanced Interfacing Techniques for Sensors. Smart Sensors, Measurement and Instrumentation; George, B., Roy, J.K., Kumar, V.J., Mukhopadhyay, S.C., Eds.; Springer International Publishing: Cham, Switzerland, 2017; pp. 191-217. [CrossRef]

26. Kai, Z.; Xiaozhang, Z. Rotor Dynamic Balance Making Use of Adaptive Unbalance Control of Active Magnetic Bearings. In Proceedings of the 2010 International Conference on Intelligent System Design and Engineering Application, Changsha, China, 13-14 October 2010; Volume 2, pp. 347-350. [CrossRef] 